\title{
Quantitation of Permethylated $N$-Glycans through Multiple-Reaction Monitoring (MRM) LC-MS/MS
}

\author{
Shiyue Zhou, Yunli Hu, Janie L. DeSantos-Garcia, Yehia Mechref
}

Department of Chemistry and Biochemistry, Texas Tech University, Lubbock, TX 79409, USA

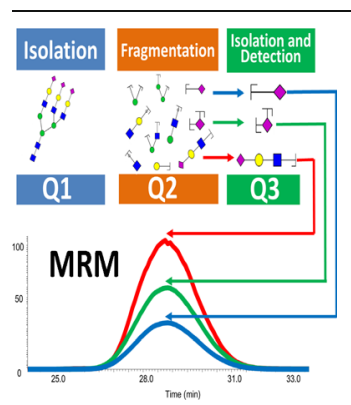

\begin{abstract}
The important biological roles of glycans and their implications in disease development and progression have created a demand for the development of sensitive quantitative glycomics methods. Quantitation of glycans existing at low abundance is still analytically challenging. In this study, an N-linked glycans quantitation method using multiple-reaction monitoring (MRM) on a triple quadrupole instrument was developed. Optimum normalized collision energy (CE) for both sialylated and fucosylated $N$-glycan was determined to be $30 \%$, whereas it was found to be $35 \%$ for either fucosylated or sialylated N-glycans. The optimum CE for mannose and complex type $\mathrm{N}$-glycan was determined to be $35 \%$. Additionally, the use of three transitions was shown to facilitate reliable quantitation. A total of $88 \mathrm{~N}$-glycan compositions
\end{abstract} in human blood serum were quantified using this MRM approach. Reliable detection and quantitation of these glycans was achieved when the equivalence of $0.005 \mu \mathrm{L}$ of blood serum was analyzed. Accordingly, $N$-glycans down to the 100th of a $\mu \mathrm{L}$ level can be reliably quantified in pooled human blood serum, spanning a dynamic concentration range of three orders of magnitude. MRM was also effectively utilized to quantitatively compare the expression of $\mathrm{N}$-glycans derived from brain-targeting breast carcinoma cells (MDA-MB-231BR) and metastatic breast cancer cells (MDA-MB-231). Thus, the described MRM method of permethylated $\mathrm{N}$-glycan enables a rapid and reliable identification and quantitation of glycans derived from glycoproteins purified or present in complex biological samples.

Keywords: Glycans, Permethylation, MRM-LC-MS, LC-MSMS, Quantitation

Received: 2 September 2014/Revised: 9 November 2014/Accepted: 9 November 2014/Published Online: 20 February 2015

\section{Introduction}

$\mathrm{G}$ lycosylation is currently recognized as one of the most common post-translational modifications (PTMs) of proteins. This PTM plays an important role in biological processes, such as cell recognition, cell-cell interaction, immune surveillance, and cell division and adhesion [1-6]. Glycosylation changes may reflect the aberrant biological activities that indicate pathologic alterations. Thus, glycans and glycoproteins can be utilized as potential therapeutic and diagnosis assets.

Accordingly, qualitative and quantitative glycomic information has been deemed necessary to understand the biological roles

Shiyue Zhou, Yunli Hu and Janie L. DeSantos-Garcia contributed equally to this work.

Electronic supplementary material The online version of this article (doi:10.1007/s13361-014-1054-1) contains supplementary material, which is available to authorized users.

Correspondence to: Yehia Mechref; e-mail: yehia.mechref@ttu.edu of glycans in biological systems. Observed differences could subsequently permit a better understanding of the biological attributes of glycan structures. Mass spectrometry (MS) is currently utilized in glycan quantitation because of its high sensitivity [7-9]. MS-based quantitation methods can be classified into two main categories: label-free or isotopic labeling approaches $[10,11]$.

The label-free approaches have been widely used in glycan quantitation studies. The $\mathrm{N}$-glycans' relative intensities of several runs were compared for quantitation purpose. Several derivatization methods, including reductive amination [1214], permethylation [15-24], and peracetylation [25, 26] have been employed to address the weak ionization efficiency of native glycans in MS sources.

Isotopic labeling of glycans is attained through chemical derivatization such as reductive amination [27-33], permethylation [34-36], metabolic incorporation of stable isotope tags [37, 38], and introduction of isotope labels via enzymatic reactions [39]. These methods are facilitating the monitoring of glycan variations between different samples in a single mass spectrometric analysis. 
The most reliable MS quantitation is selected reaction monitoring (SRM) and multiple reaction monitoring (MRM). These are MS modes with the first quadrupole (Q1) filters the specific $\mathrm{m} / \mathrm{z}$ values of the precursor ion, and the third quadrupole (Q3) filters the corresponding fragments of the precursor (ion transitions), whereas the second quadrupole (Q2) is a collision cell. Instead of quantifying ions using full MS scan, the quantitation of SRM and MRM is based on ion transitions, which can significantly enhance the signal-to-noise ratios for the detection of low abundant components. SRM is a targeted technique that selects a single transition for simultaneous identification and quantitation, which has been introduced for quantitation of proteins [40], glycopeptides [41], and glycans [42] in complex samples. In MRM mode, multiple transitions were selected, thus allowing more precise analytical quantitation compared with SRM. MRM has been used for glycopeptide quantitation [43-47]. The first use of MRM for the analysis of glucose tetrasaccharide was reported in 2003 [48]. Recently, another two MRM quantitation studies were published for the analysis of reduced native glycan [49] and 1-phenyl-3-methyl-5-pyrazolone (PMP) labeled glycan [50]. However, until now, this method has not been exploited for permethylated $N$-glycan analysis.

Herein, we describe MRM for quantitation of permethylated $\mathrm{N}$-glycans. The effectiveness and the analytical precision of MRM rely on transitions. Therefore, it is critical to choose representative transitions and optimize collision energy for efficient fragmentation. The optimization of transitions was performed using permethylated $N$-glycans derived from model glycoproteins, such as ribonuclease B, fetuin, and porcine thyroglobulin. The number of transitions, precursor ions selection, and normalized collision energy were optimized to obtain effective and reliable MRM quantitation. Glycomic profiles of complex biological samples, such as human blood serum and cancer cell lines, were also acquired using the described MRM method to evaluate the ability to quantify glycans at low abundances. The linear concentration dynamic range and detection limits of $\mathrm{N}$-glycans derived from model glycoproteins and different biological samples were also investigated.

\section{Experimental}

\section{Materials and Reagents}

Model glycoproteins, ribonuclease B (RNase B) from bovine pancreas, fetuin from bovine serum, porcine thyroglobulin (PTG), pooled human blood serum (HBS), borane-ammonia complex, sodium hydroxide beads, dimethyl sulfoxide (DMSO), iodomethane, trifluoroacetic acid, and formic acid were purchased from Sigma-Aldrich (St. Louis, MO, USA). Cell line MDA-MB-231 was purchased from American Type Culture Collection (ATCC, Manassas, VA, USA), and MDAMB-231BR was a generous gift from Dr. Paul Lockman (Texas Tech Health Sciences Centers, School of Pharmacy, Amarillo, TX, USA). PNGase F, $10 \times$ G7 reaction buffer $(50 \mathrm{mM}$ sodium phosphate buffer, $\mathrm{pH} 7.5), 10 \times$ glycoprotein denaturing buffer (50 mM sodium phosphate buffer, $\mathrm{pH} 7.5,5 \% \mathrm{SDS}$,
0.4 $\mathrm{M}$ dithiothreitol), and nonionic NP-40 detergent were acquired from New England Biolabs (Ipswich, MA, USA). High performance liquid chromatography (HPLC) grade methanol, isopropanol, and acetic acid were procured from Fisher Scientific (Pittsburgh, PA, USA). Acetonitrile (ACN) was obtained from J.T. Baker (Phillipsburg, NJ, USA). HPLC grade water and sodium hydroxide were purchased from Mallinckrodt Chemicals (Phillipsburg, NJ, USA).

\section{Release, Purification, and Reduction of N-glycans from Model Glycoproteins and Pooled Human Blood Serum}

A $1-\mu \mathrm{g} / \mu \mathrm{L}$ aliquot of RNase B and $10 \mu \mathrm{g} / \mu \mathrm{L}$ stock solutions of fetuin and PTG were prepared. A 25- $\mu$ g aliquot of RNase B, $100-\mu \mathrm{g}$ aliquot of fetuin, $100-\mu \mathrm{g}$ aliquot of PTG, and a $50-\mu \mathrm{L}$ aliquot of HBS were taken from stock solutions. A $20-\mu \mathrm{L}$ aliquot of phosphate-buffered saline (PBS) $(50 \mathrm{mM}$ sodium phosphate, $100 \mathrm{M}$ sodium chloride, $\mathrm{pH} 7.5$ ) was added to each sample. Samples were then incubated at a $60^{\circ} \mathrm{C}$ in a water bath (Thermo Scientific, Pittsburgh, PA, USA) for $1 \mathrm{~h}$. A 2.4- $\mu \mathrm{L}$ aliquot of PNGase F diluted in $10 \times \mathrm{G} 7$ reaction buffer was added to each sample. Samples were then incubated overnight at $37^{\circ} \mathrm{C}$ in a water bath. Next, released glycans were purified to remove salts and impurities using drop-dialysis for $18 \mathrm{~h}$ on a 500-1000 Da cut-off molecular weight cellulose ester dialysis membrane (Spectrum Laboratories, Rancho Dominguez, CA, USA). Reduction of $N$-glycans was achieved by adding a 10 $\mu \mathrm{L}$ aliquot of a $10 \mu \mathrm{g} / \mu \mathrm{L}$ ammonium-borane complex solution prepared water and. Samples were placed in $60^{\circ} \mathrm{C}$ water bath for $1 \mathrm{~h}$. A $10-\mu \mathrm{L}$ aliquot of $5 \%$ acetic acid aqueous solution was added to each sample and dried under vacuum. Finally, a 300$\mu \mathrm{L}$ aliquot of HPLC grade methanol was added to each sample and dried under vacuum. This was repeated until no more borate salt was visible.

\section{Cell Lysis, Protein Assay, Denaturation, Release, Purification, and Reduction of N-Glycans from Cell Lines}

Breast cancer cell lines MDA-MB-231 and its brain targeting subline MDA-MB-231BR were initially suspended in $100-\mu \mathrm{L}$ aliquots of PBS. The suspensions were then sonicated in iced water for $60 \mathrm{~min}$. A $5-\mu \mathrm{L}$ aliquot of the sonicated cells was subjected to BSA protein assay as suggested by the vendor (Fisher Scientific, Pittsburgh, PA, USA) to determine protein concentrations. Next, the proteomes of lysates were denatured in $80^{\circ} \mathrm{C}$ water bath (Thermo Scientific) for $1 \mathrm{~h}$. These samples were then cooled to room temperature prior to the addition of a 2.4- $\mu \mathrm{L}$ aliquot of a $10 \times$ diluted PNGase F solution (50 U, New England Biolabs). Enzymatic digestion was allowed to proceed at $37^{\circ} \mathrm{C}$ in a water bath for $18 \mathrm{~h}$. Glycans released from the proteome of cell-line samples were purified by charcoal spincolumn as previously described [19, 22, 51]. The columns were washed with a $400-\mu \mathrm{L}$ aliquot of $100 \% \mathrm{ACN}$ and a $400-\mu \mathrm{L}$ aliquot of $85 \%$ ACN with $0.1 \%$ TFA 3 -times. The spin-column was then conditioned with a $400-\mu \mathrm{L}$ aliquot of $5 \% \mathrm{ACN}$ with 
$0.1 \%$ TFA twice. Next, sample volumes were adjusted to $400 \mu \mathrm{L}$ using $5 \% \mathrm{ACN}$ with $0.1 \%$ TFA and washed 4-times with a $400-\mu \mathrm{L}$ aliquot of $5 \%$ ACN with $0.1 \%$ TFA. Glycans were then eluted using a $400-\mu \mathrm{L}$ aliquot of $40 \%$ ACN with $0.1 \%$ TFA. Samples were dried under vacuum (Labconco, Kansas City, MO, USA). Finally, glycans were reduced using borane-ammonium complex as described above.

Solid-Phase Permethylation of N-Glycans Released from Model Glycoproteins, Pooled Human Blood Serum, and Cell Lines

The N-glycans derived from model glycoproteins, human blood serum samples, and cell lines were derivatized by solid-phase permethylation as previously described [17, 18, 52]. Briefly, spin columns (Harvard Apparatus, Holliston, MA, USA) were filled to a $3-\mathrm{cm}$ height with $\mathrm{NaOH}$ beads. The columns were then placed in Eppendorf tubes prior to the addition of a $200-\mu \mathrm{L}$ aliquot of DMSO. The samples were then centrifuged at $1.6 \mathrm{k} \mathrm{rpm}$. This was repeated twice.

Released $\mathrm{N}$-glycans from RNase B, fetuin, PTG, and HBS were resuspended in $30 \mu \mathrm{L}$ high purity DMSO, whereas MDAMB-231BR cell line and MDA-MB-231 cell line were resuspended in $15 \mu \mathrm{L}$ high purity DMSO. A $1.2-\mu \mathrm{L}$ aliquot of water was added to all samples and mixed prior to the addition of $20 \mu \mathrm{L}$ methyl iodide. The conditioned spin columns were transferred to new $2 \mathrm{~mL}$ Eppendorf tubes, and the sample mixture was applied to the columns and allowed to sit for $25 \mathrm{~min}$, then centrifuged at $1.6 \mathrm{k} \mathrm{rpm}$ for $1 \mathrm{~min}$, followed by another 15-min incubation after the addition of a second aliquot of $20-\mu \mathrm{L}$ methyl iodide. Then the columns were centrifuged again at $1.6 \mathrm{k} \mathrm{rpm}$ for $1 \mathrm{~min}$. Permethylated $N$-glycans were eluted using a $50-\mu \mathrm{L}$ aliquot of $\mathrm{ACN}$ twice. Then the samples were dried under vacuum for online purification. The samples were resuspended in $20 \%$ aqueous $\mathrm{ACN}$ solution to obtain a series of dilutions from 1 to $1000 \mathrm{ng} / \mu \mathrm{L}$ for each model glycoproteins, and a dilution ranged from 0.005 to $0.5 \mu \mathrm{g} / \mu \mathrm{L}$ for blood serum. The final concentration of MDA-MB-231BR cell line and MDA-MB-231 after normalization was $0.47 \mu \mathrm{g}$ of protein $/ \mu \mathrm{L}$ of solution.

\section{LC Separation Conditions}

Reduced and permethylated glycans were separated by nanoLC system (Ultimate 3000; Dionex, Sunnyvale, CA, USA) on a reverse phase Acclaim PepMap capillary column $(150 \mathrm{~mm} \times$ $75 \mu \mathrm{m}$ i.d.) packed with $100 \AA \mathrm{C}_{18}$ bounded phase (Dionex). The mobile phases were composed of solvent A ( $98 \%$ HPLCgrade water, $2 \% \mathrm{ACN}, 0.1 \% \mathrm{FA})$ and solvent $\mathrm{B}(98 \% \mathrm{ACN}$, $2 \%$ HPLC-grade water $0.1 \%$ FA). Prior to injection into the column, the samples were trapped on an Acclaim PepMap trapping column and washed for $10 \mathrm{~min}$ at a flow rate of $3 \mu \mathrm{L} / \mathrm{min}$ using solvent $\mathrm{A}$. The separation of reduced and permethylated glycans was attained with a gradient of solvent B increasing from $20 \%$ to $38 \%$ over $11 \mathrm{~min}$, from $38 \%$ to $45 \%$ over the next $22 \mathrm{~min}$, then a fast ramp to $90 \% \mathrm{~B}$ in $3 \mathrm{~min}$, stay at $90 \%$ for $4 \mathrm{~min}$, then decreased from $90 \%$ to $20 \%$ in $1 \mathrm{~min}$, and held at 20\% B for the next $9 \mathrm{~min}$. The separation was achieved at a $350-\mathrm{nL} / \mathrm{min}$ flow rate.

\section{MS Data Acquisition}

Thermo Scientific TSQ Vantage triple quadrupole mass spectrometer was employed in this study. Data-dependent acquisition mode (DDA) with two scan events was performed in positive ion mode. The first event is a full MS scan at range of $300-1500 \mathrm{~m} / \mathrm{z}$ in $\mathrm{Q}_{3}$ with a scan time of $0.7 \mathrm{~s}$ and peak width of 0.7 FWHM. Data-dependent scan occurs for the second scan event where the five most intense ions from scan event one were subsequently selected and subjected to MS/MS. Precursor ion scan (PIS) has six scan events with each of them containing a product mass. The peak width was set to 0.7. MRM experiment was performed with a peak width of $0.7 \mathrm{FWHM}$, and scan time of $0.7 \mathrm{~s}$ for $500-1500 \mathrm{~m} / \mathrm{z}$ scan range. The normalized CE values ranged from $30 \%$ to $45 \%$ in order to determine the most effective condition. Peak areas were determined by using Xcalibur software (Thermo Scientific).

\section{Data Evaluation}

Extracted-ion chromatograms from the full MS scans were generated using Xcalibur Qual Browser (Thermo Fisher Scientific). Tandem mass spectra corresponding to glycan ions were manually annotated. The three most intense diagnostic ions assigned from MSMS spectrum were used as transitions. The reconstructed precursor ions from PIS were utilized to confirm diagnostic fragment ions (transitions). In MRM mode, three transitions were summed for each precursor ion to generate extracted ion chromatogram in Xcalibur Qual Browser. Peak areas were used for glycan quantitation.

\section{Results and Discussion}

The identification and quantitation of permethylated $\mathrm{N}$-Glycans workflow are summarized in Figure 1. DDA mode, which selects several ions of interest for subsequent MS/MS analysis was first used to obtain structural information of glycans within each sample: RNaseB, Fetuin, PTG, HBS, and cell lines (Figure 1a). The full MS scan under the retention time of $20 \mathrm{~min}$ is shown on Figure 1b. The MSMS scan (Figure 1c) provides the fragmentation information of the precursor ions. Three most intense fragments were selected as transitions. PIS (Figure 1d) was further applied to confirm that the selected transitions are a true representation of the precursor ions. For example, the selected product ion at $344 \mathrm{~m} / \mathrm{z}$ associated the precursor ion at an $\mathrm{m} / \mathrm{z}$ value of 929.5 confirms that it is a fragment of the bi-antennary disialylated glycan. The transitions that are confirmed through PIS were then used in MRM quantitation (Figure 1e).

Selecting adequate ion transitions for each glycan structure is a critical component of this methodology. A series of tests were performed using one, two, or three transitions to determine the optimized transitions number. As shown in 


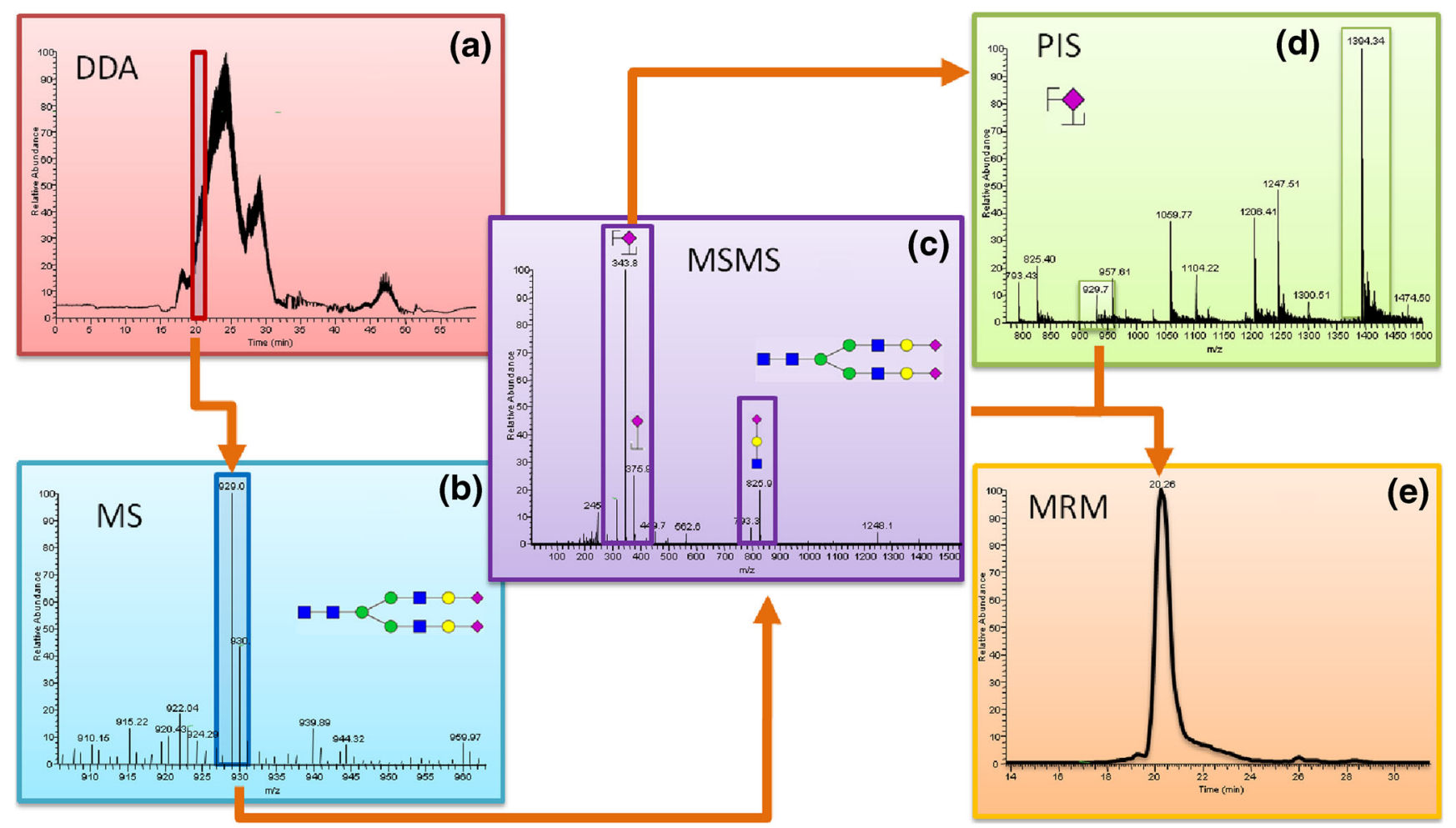

Figure 1. Workflow summarizing the analysis of permethylated $N$-glycans derived from RNase B, Fetuin, PTG, and pooled human blood serum on triple quadrupole mass spectrometer (a) DDA chromatogram, (b) precursor ion MS scan, (c) MSMS scan for selected PIS, (d) selected transitions are applied to PIS, (e) selected precursor ions and transitions are applied to MRM

Supplementary Figure S1 in Supporting Information, the intensity of $\mathrm{N}$-glycans derived from RNase B increased with the increasing of the transition number. The optimal amount of transitions for quantitation was three, as shown by the highest intensity obtained for all analytes. Similar results were reported for the MRM quantitation of glycopeptides [47]. Also, increasing characteristic fragment for a specific glycan structure allows reducing the interference from other $\mathrm{N}$-glycans and increase the selectivity of the method. However, including too many transitions for one precursor ion would increase duty cycle, resulting in decreased number of detected glycans. Hence we set the transition number to be three to achieve best quantitation results.

Representative transitions selection is another importance component of MRM quantitation. The $\mathrm{N}$-glycan structures were divided into high mannose structure, complex structure hybrid structure (Supplementary Table S1 in Supporting Information). For each group, there are several characteristic fragments. For example, hexose fragments are commonly observed in the case of high mannose structure at $\mathrm{m} / \mathrm{z}$ values of $110\left({ }^{0,2} \mathrm{X}\right), 230$ (YZZ), 187(BZ), 262(YZ), and 294 (Y). The ion transitions selected for complex structures with and without fucose were 260 (HexNac), 228( $\left.\mathrm{HexNAc}-\mathrm{CH}_{3} \mathrm{OH}\right)$, and 196 (HexNAc- $\left.2 \mathrm{CH}_{3} \mathrm{OH}\right)$. With the increase of molecular weight, more Hex-HexNAc $(m / z=432$ and 460$)$ fragments are likely to be formed. In the case of sialic acid glycans, characteristic fragment ions containing sialic acid were observed at $\mathrm{m} / \mathrm{z}$ values of 344,376 , and 825 . Similar fragment patterns were also reported in previous studies involving permethylated $\mathrm{N}$ - glycans [53-56]. For glycans that have fucose and sialic acid, the sialic acid characteristic ions at $\mathrm{m} / \mathrm{z}$ values of 312,344 , and 376 were applied as transitions. The fact that the presence of fucose does not affect the transition selection might be because the fucosyl linkage is much weaker than the other glycosidic linkages. Thus, the diagnostic ions with fucosylation are usually not observed [56, 57]. The transitions of hybrid $N$-glycans also depend on the subtype of the structure. For example, hybrid $N$-glycans with sialic acid are likely to form fragment ions at $m / z$ values of 344,376 , and 825 , whereas the transitions of hybrid $N$-glycans without sialic acid are similar to those of complex $\mathrm{N}$-glycans. Accordingly, the transitions selected enable the identification of the glycan type. (i.e., complex-, high mannose-, hybrid-type, sialylated, and fucosylated). In this study, we only focused on classifying the glycans into groups. The selected transitions do not enable the identification of structural isomers. However, we are currently working on optimizing the chromatographic conditions to prompt isomeric separations that will enable the identification and quantification of glycan isomers. A challenge, which is limiting the selection of transitions that enable the identification of isomers, is the fact that MS/MS alone might not be sufficient for linkage identification since a limited number of cross-ring fragments is observed in collision induced dissociation (CID). $\mathrm{MS}^{\mathrm{n}}$ is required for comprehensive structure elucidation of glycan isomers. Additionally, choosing transitions that are linkagespecific for quantification of $\mathrm{N}$-glycans is not feasible because of the intensities of such fragment is low. 
Precursor ion selection is another important factor for MRM quantitation. Glycans are likely to form multiple charge state ions and adducts. Also, the intensity of most abundance precursor ion was representative of the intensities of all adducts [58]. It is critical to choose the most intense ion adducts as precursor ions. For example, the high mannose glycans are more likely to form ammonium adducts instead of protonated adducts. The distribution of high mannose composition derived from RNase B will not be representative of the native form if the protonated ions were used as precursor ions. Therefore, defining the most intense ions as precursor ions is required.

Optimum CE for each structure was obtained by performing through a series of different normalized $\mathrm{CE}$ values spanning from $30 \%$ to $45 \%$. At a CE value of $30 \%$, the majority of $N$ glycans except complex glycans with fucose and sialic acid did not attain complete fragmentation and depicted low intensities. The optimal CE value was determined to be $35 \%$ for mannose, complex type, and hybrid type glycans (Supplementary Figure S2). The phenomena observed for the fucosylated and sialylated glycans can be attributed to the weak glycosidic linkages. At a CE value of $45 \%$, ion fragments were further fragmented, which resulted in a loss of fragment integrity and caused an overall decreased in intensity.

$N$-glycans derived from model glycoproteins were subjected to triple quadrupole MS using MRM mode to evaluate the quantitation possibility. Different concentrations of permethylated RNase B glycans were obtained from a serial dilution. As the injection amount of reduced and permethylated RNase B went as low as $1 \mathrm{ng}$, all five peaks associated with RNase B were detected. The sensitivity of RNase B is higher

(a)

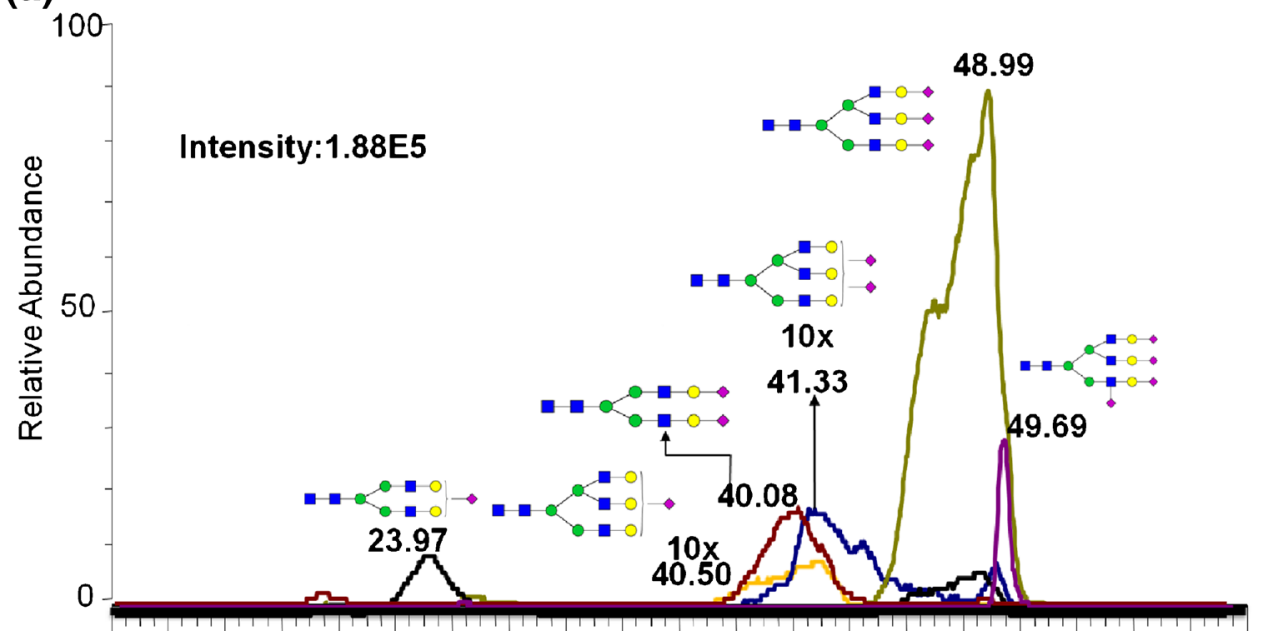

1012141618202224262830323436384042444648505254565860

Time (min)

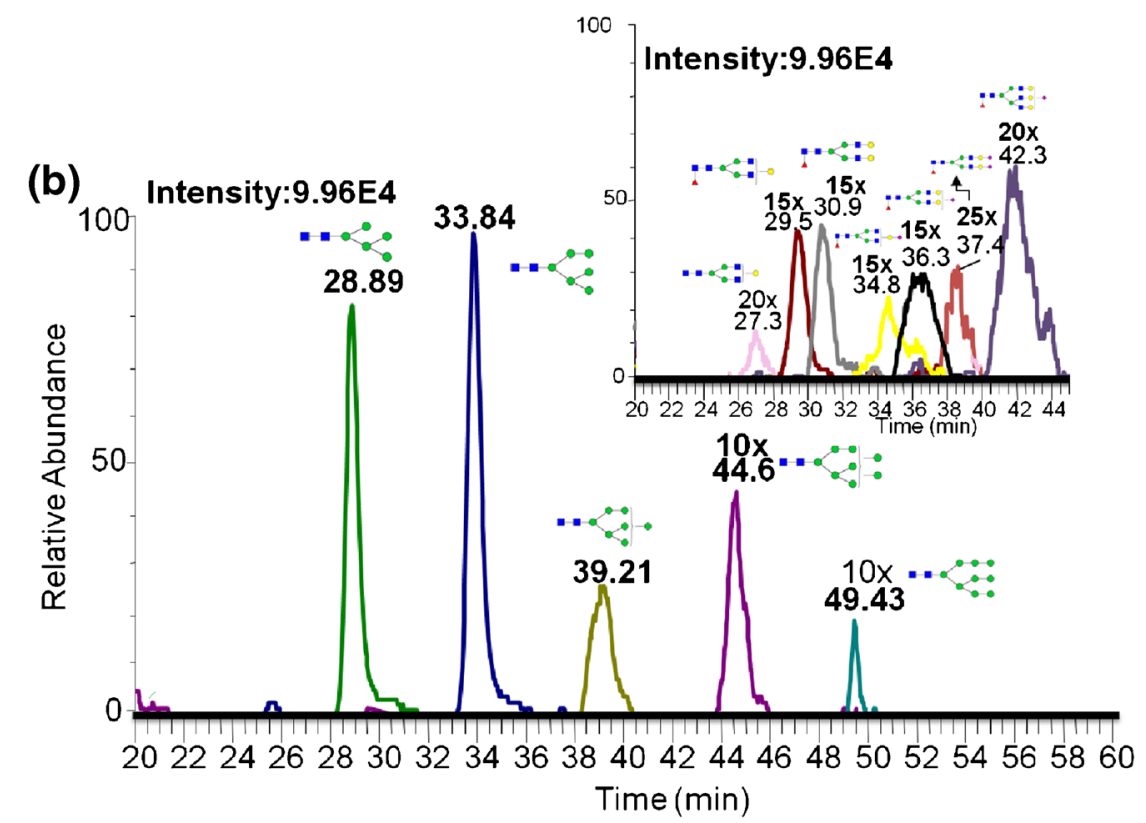

Figure 2. Extracted ion chromatograms of permethylated $N$-glycans derived from (a) 15 ng fetuin, (b) 25 ng PTG 
than our previous reported work of permethylated $\mathrm{N}$-glycan analyzed by the data-dependent mode of the Orbitrap mass spectrometer [59]. The high sensitivity of this method might be attributed to the two-stage mass filter of MRM, which decreases the chemical noise [60]. In another study, Zhang et al. [42] used LC-SRM with aniline-labeled RNase B glycans and detected all five peaks at $50 \mu \mathrm{g}$. The sensitivity attained in the case of permethylated glycans is substantially higher than that described for aniline-labeled $\mathrm{N}$-glycans derived from $\mathrm{RN}$ ase $\mathrm{B}$. This might be due to permethylation, which is known to enhance ionization efficiency of glycans as a result of increased hydrophobicity originating from the introduction of methyl groups. Moreover, increased sensitivity in our strategy can also be attributed to MRM methodology using representative fragment ions as transitions for a certain type of $\mathrm{N}$-glycan. Supplementary Table $\mathrm{S} 2$ depicts the linear dynamic range of $\mathrm{N}$-glycans derived from RNase $\mathrm{B}$. The $\mathrm{R}^{2}$ of five structures is higher than 0.99 at the range of 1 to $100 \mathrm{ng}$. The instrument ion detectors were saturated when the injection amount of Man 5 and Man 6 derived from RNase B exceeded $100 \mathrm{ng}$.
A serial dilution of $\mathrm{N}$-glycans derived from fetuin and PTG glycoproteins were also used to investigate the quantitation ability of MRM. The linear dynamic range of fetuin was from 15 to $100 \mathrm{ng}$ with an $\mathrm{R}^{2}$ value better than 0.95 (Supplementary Table S2). Six $N$-glycans derived from fetuin were all detected at sample injection amount as low as $15 \mathrm{ng}$ of the glycoprotein (Figure 2a).

A similar trend was also observed for permethylated $\mathrm{N}$ glycans derived from PTG. Figure $2 \mathrm{~b}$ depicts the detected $\mathrm{N}$ glycans derived from PTG at an injection amount of $25 \mathrm{ng}$ of the glycoprotein. The correlation coefficients $\mathrm{R}^{2}$ were better than 0.95 at a linear dynamic concentration range of 25 to $500 \mathrm{ng}$. A wide linear dynamic range for N-glycan quantitation in MRM allows the reliable analysis of disease and disease free biological samples, in which the intensities of certain glycan structures could vary within an order of magnitude.

The developed method was further validated using complex biological samples, such as HBS. Five different concentrations of HBS glycans were analyzed by MS. There are $88 \mathrm{~N}$-glycans detected at an injection equivalent to permethylated glycans

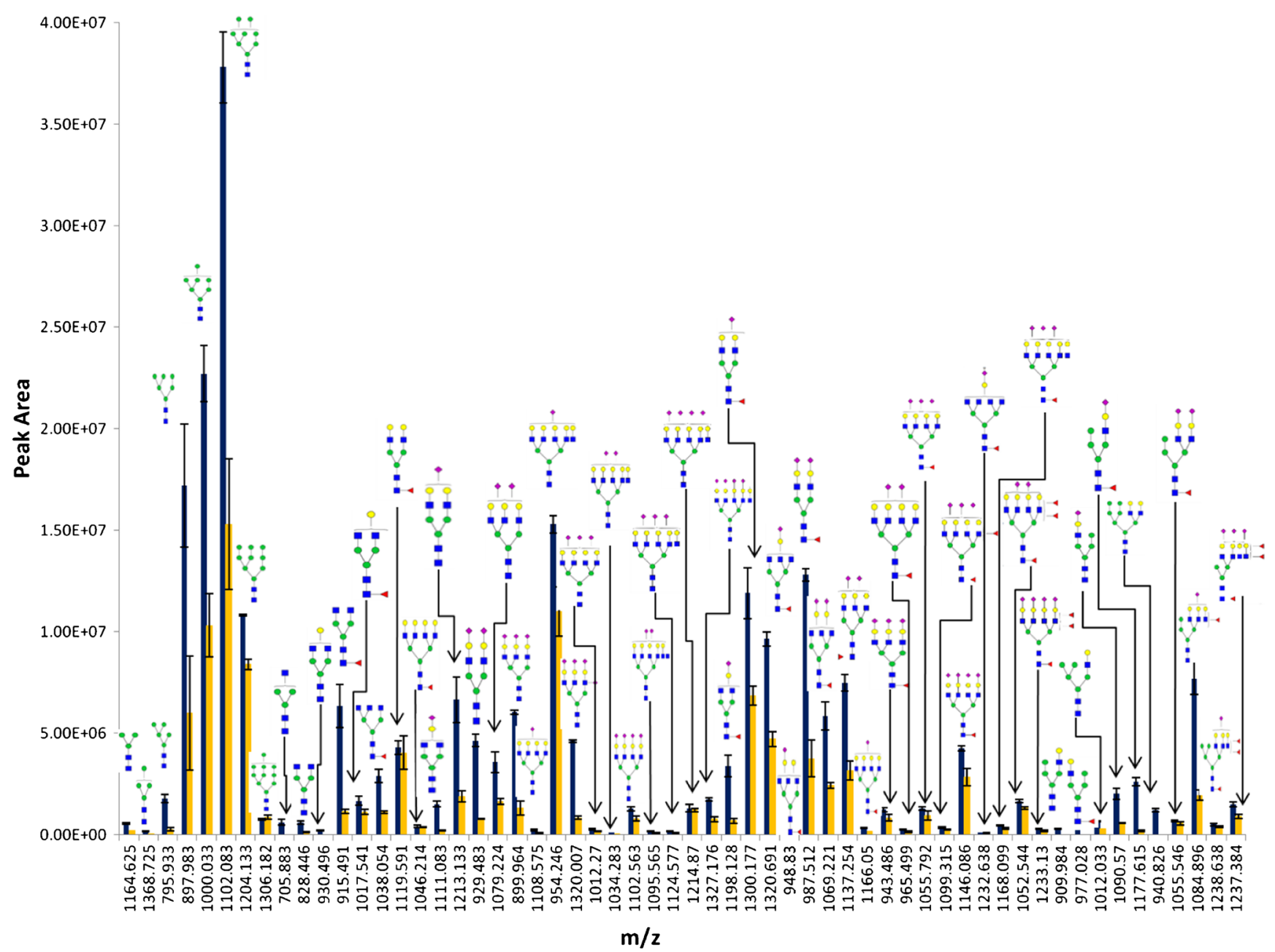

Figure 3. Comparison of the extracted ion chromatograms of permethylated $N$-glycans derived from MDA-MB-231BR cell line (blue), MDA-MB-231 cell line (yellow) through MRM LC-MS 
derived from a $0.1-\mu \mathrm{L}$ aliquot of HBS. Even at the injection amount of $0.005 \mu \mathrm{L}$ HBS, 55 glycan compositions were detected (Supplementary Table S3). Previously, only 73 permethylated $\mathrm{N}$-glycans were observed using LTQ Orbitrap Velos mass spectrometer [61]. The high sensitivity and a larger concentration dynamic range observed might be due to the twostage mass filtering [60]. The MRM mode platform in a triple quadrupole MS provides targeted analysis that significantly decreases the chemical noise that is commonly associated with mass spectrometric detection. In another study, the MRM mode enables the detection of 42 aniline labeled $N$-glycans derived from rabbit serum [42]. The higher number of detected $N$ glycans in this study might be due to the different species used and higher sensitivity prompted by permethylation.

The aforementioned targeted methodology permits highly sensitive identification and quantitation of glycans from glycoproteins and HBS. This method is further applied to profiling $N$-glycan derived from two different cancer cells lines, MDA-MB-231 and MDA-MB-231BR. MDA-MB-231 is a breast cancer cell line capable of metastasizing anywhere, and MDA-MB-231BR is the brain seeking clone of MDA-MB-231. Since the mechanism of cancer cells crossing the blood-brain-barrier (BBB) is still not completely understood, establishing glycomic profiles of cancer cells could be of significant importance to better understand the role that they play in enhancing breast cancer cell's ability to cross $\mathrm{BBB}$. The comprehensive transition list and optimized CE were applied in the quantitation of permethylated glycans derived from the glycoproteins associated with cell lines. The relative intensities of glycans derived from MDA-MB-231 and MDA-MB-231BR are shown in Figure 3. In total, $58 \mathrm{~N}$-glycans were detected from MDA-MB-231BR and $53 \mathrm{~N}$-glycans were detected for the MDAMB-231 (Figure 3, Supplementary Table S4). The number of $N$ glycans detected using MRM is slightly higher compared with that attained using the LTQ Orbitrap Velos mass spectrometer (55 $\mathrm{N}$-glycans were detected for MDA-MB-231BR and $50 \mathrm{~N}$-glycan compositions were observed from MDA-MB-231, data not shown). Thus, MRM provides greater quantitative information for the lower abundant $N$-glycans compared with the LTQ Orbitrap Velos. The average \%CV of peak areas of $\mathrm{N}$-glycans derived from MDA-MB-231BR and MDA-MB-231 was 10.3\% and $17 \%$, respectively. The low $\% \mathrm{CV}$ indicates the reproducibility of this quantitation strategy. The average $\% \mathrm{CV}$ of electrospray ionization (ESI) is around $20 \%$. The overall low $\% \mathrm{CV}$ indicates high reproducibility of this strategy for $N$-glycan quantitation.

\section{Conclusions}

The aforementioned method has been shown to be a viable strategy for quantitation of $N$-linked glycans in simple and complex mixtures. The optimized CE conditions for MRM mode were set for at $30 \%$ for mannose and complex type, $35 \%$ for sialylated and fucosylated $\mathrm{N}$-glycans. In addition, three high intensity and characteristic ions were chosen. These conditions were first validated using $\mathrm{N}$-glycans derived from model glycoproteins, then were applied to HBS and then the cell lines.
Utilizing this newly developed method, linear dynamic range expanding over three orders of magnitude with high reproducibility was established. This approach allows for greater sensitivity and reproducibility in complex biological samples, including HBS and cell lines. A comprehensive list of permethylated glycans and their optimum ion transitions are compiled in this study that could be used in future studies to quantify glycomic changes associated with perturbed biological systems.

\section{Acknowledgments}

The authors acknowledge funding of this work by CPRITRP130624 and NIH-GM (1R01GM093322-01). The authors express thanks to Dr. Paul Lockman and Dr. Quentin Smith from TTU/HSC, School of Pharmacy in Amarillo for providing cell line MDA-MB-231BR.

\section{References}

1. Varki, A.: Biological roles of oligosaccharides: all of the theories are correct. Glycobiology 3, 97-130 (1993)

2. Dwek, R.A.: Glycobiology - toward understanding the function of sugars. Chem. Rev. 96, 683-720 (1996)

3. Rudd, P.M., Wormald, M.R., Stanfield, R.L., Huang, M., Mattson, N., Speir, J.A., DiGennaro, J.A., Fetrow, J.S., Dwek, R.A., Wilson, I.A.: Roles for glycosylation of cell surface receptors involved in cellular immune recognition. J. Mol. Biol. 293, 351-366 (1999)

4. Dennis, J.W., Granovsky, M., Warren, C.E.: Protein glycosylation in development and disease. BioEssays 21, 412-421 (1999)

5. Lowe, J.W., Marth, M.: A genetic approach to Mammalian glycan function. Annu. Rev. Biochem. 72, 643-691 (2003)

6. Rudd, P.M., Woods, R.J., Wormald, M.R., Opdenakker, G., Downing, A.K., Campbell, I.D., Dwek, R.A.: The effects of variable glycosylation on the functional activities of ribonuclease, plasminogen, and tissue plasminogen activator. Biochim. Biophys. Acta 1248, 1-10 (1995)

7. Dell, A., Morris, H.R.: Glycoprotein structure determination by mass spectrometry. Science 291, 2351-2356 (2001)

8. H. Geiser, C. Silvescu, V. Reinhold, Separation methods in proteomics. CRC Press, Boca Raton, Florida (2006)

9. Mechref, Y., Novotny, M.: Structural investigations of glycoconjugates at high sensitivity. Chem. Rev. 102, 321-369 (2002)

10. Ruhaak, L.R., Zauner, G., Huhn, C., Bruggink, C., Deelder, A.M., Wuhrer, M.: Glycan labeling strategies and their use in identification and quantification. Anal. Bioanal. Chem. 397, 3457-3481 (2010)

11. Mechref, Y., Hu, Y., Desantos-Garcia, J.L., Hussein, A., Tang, H.: Quantitative glycomic strategies. Mol. Cell Proteomics 12, 874-884 (2012)

12. Ruhaak, L.R., Huhn, C., Waterreus, W.J., de Boeer, A.R., Neususs, C., Hokke, C.H., Deelder, A.M., Wuhrer, M.: Hydrophilic interaction chromatography-based high-throughput sample preparation method for $\mathrm{N}$ glycan analysis from total human plasma glycoproteins. Anal. Chem. 80, 6119-6126 (2008)

13. Gil, G.C., IIif, B., Cerny, R., Velander, W.H., Van Cott, K.E.: High throughput quantification of $\mathrm{N}$-glycans using one-pot sialic acid modification and matrix assisted laser desorption ionization time-of-flight mass spectrometry. Anal. Chem. 82, 6613-6620 (2010)

14. Klapoetke, S., Zhang, J., Becht, S., Xuelin, G., Ding, X.: The evaluation of a novel approach for the profiling and identification of N-linked glycan with a procainamide tag by HPLC with fluorescent and mass spectrometric detection. J. Pharm. Biomed. Anal. 53, 315-324 (2010)

15. Ciucanu, I., Kerek, F.: A simple and rapid method for the permethylation of carbohydrates. Carbohydr. Res. 131, 209-217 (1986)

16. Ciucanu, I., Costello, C.E.: Elimination of oxidative degradation during per-Omethylation of carbohydrates. J. Am. Chem. Soc. 125, 16213-16219 (2003)

17. Kang, P., Mechref, Y., Klouckova, I., Novotny, M.V.: Solid-phase permethylation of glycans for mass spectrometric analysis. Rapid Commun. Mass Spectrom. 19, 3421-3428 (2005) 
18. Kang, P., Mechref, Y., Novotny, M.V.: High-throughput solid-phase permethylation of glycans prior to mass spectrometry. Rapid Commun. Mass Spectrom. 22, 721-734 (2008)

19. Kyselova, Z., Mechref, Y., Al Bataineh, M.M., Dobrolecki, L.E., Hickey, R.J., Vinson, J., Sweeney, C.J., Novotny, M.V.: Alterations in the serum glycome due to metastatic prostate cancer. J. Proteome Res. 6, 1822-1832 (2007)

20. Goldman, R., Ressom, H., Varghese, R., Goldman, L., Bascug, G., Loffredo, C., Abdel-Hamid, M., Gouda, I., Abo-Elkhir, S., Kyselova, Z., Mechref, Y., Novotny, M.V.: Detection of hepatocellular carcinoma using glycomic analysis. Clin. Cancer Res. 15, 1808-1813 (2009)

21. Kyselova, Z., Mechref, Y., Kang, P., Goetz, J.A., Dobrolecki, L.E., Sledge, G., Schnaper, L., Hickey, R.J., Malkas, L.H., Novotny, M.V.: Breast cancer diagnosis/prognosis through quantitative measurements of serum glycan profiles. Clin. Chem. 54, 1166-1175 (2008)

22. Mechref, Y., Hussein, A., Bekesova, S., Pungpapong, V., Zhang, M., Dobrolecki, L.E., Hickey, R.J., Hammoud, Z.T., Novotny, M.V.: Quantitative serum glycomics of esophageal adenocarcinoma and other esophageal disease onsets. J. Protoeme Res. 8, 2656-2666 (2009)

23. Alley, W.R.J., Vasseur, J.A., Goetz, J.A., Svoboda, M., Mann, B.F., Matei, D.E., Menning, N., Hussein, A., Mechref, Y., Novotny, M.V.: N-linked glycan structures and their expressions change in the blood sera of ovarian cancer patients. J. Proteome Res. 11, 2282-2300 (2012)

24. Jeong, H.-J., Kim, Y.-G., Yang, Y.-H., Kim, B.-G.: High-throughput quantitative analysis of total $\mathrm{N}$-glycans by matrix-assisted laser desorption/ionization time-of-flight mass spectrometry. Anal. Chem. 84, 3453-3460 (2012)

25. Dell, A.: Preparation and desorption mass spectrometry of permethyl and peracetyl derivatives of oligosaccharides. Methods Enzymol. 193, 647-660 (1990)

26. Mechref, Y., Novotny, M.V.: Structural characterization of oligosaccharides using MALDI-TOF/TOF tandem mass spectrometry. Anal. Chem. 70, 455-463 (1998)

27. Xia, B., Feasley, C.L., Sachdev, G.P., Smith, D.F., Cummings, R.D.: Glycan reductive isotope labeling for quantitative glycomics. Anal. Biochem. 387, 162-170 (2009)

28. Bowman, M.J., Zaia, J.: Tags for the stable isotopic labeling of carbohydrates and quantitative analysis by mass spectrometry. Anal. Chem. 79, 5777-5784 (2007)

29. Bowman, M.J., Zaia, J.: Comparative glycomics using a tetraplex stableisotope coded tag. Anal. Chem. 82, 3023-3031 (2010)

30. Hitchcock, A.M., Costello, C.E., Zaia, J.: Glycoform quantification of chondroitin/dermatan sulfate using an LC/MS/MS platform. Biochemistry 45, 2350-2361 (2006)

31. Hitchcock, A.M., Yates, K.E., Shortkroff, S., Costello, C.E., Zaia, J.: Optimized extraction of glycosaminoglycans from normal and osteoarthritic cartilage for glycomics profiling. Glycobiology 17, 25-35 (2006)

32. Prien, J.M., Prater, B.D., Qin, Q., Corckrill, S.L.: Mass spectrometric-based stable isotopic 2-aminobenzoic acid glycan mapping for rapid glycan screening of biotherapeutics. Anal. Chem. 82, 1498-1508 (2010)

33. Lawrence, R., Olson, S.K., Steele, R.E., Wang, L., Warrior, R., Cummings, R.D., Esko, J.D.: Evolutionary differences in glycosaminoglycan fine structure detected by quantitative glycan reductive isotope labeling. J. Biol. Chem. 283, 33674-33684 (2008)

34. Atwood III, J.A., Cheng, L., Alvarez-Manilla, G., Warren, N.L., York, W.S., Orlando, R.: Quantitation by isobaric labeling: applications to glycomics. J. Proteome Res. 7, 367-374 (2008)

35. Kang, P., Mechref, Y., Kyselova, Z., Goetz, J.A., Novotny, M.V.: Comparative glycomic mapping through quantitative permethylation and stableisotope labeling. Anal. Chem. 79, 6064-6073 (2007)

36. Hu, Y., Desantos-Garcia, J.L., Mechref, Y.: Comparative glycomic profiling of isotopically permethylated $N$-glycans by LC-ESI-MS. Rapid Commun. Mass Spectrom. 27, 865-877 (2013)

37. Orlando, R., Lim, J.-M., Atwood III, J.A., Angel, P.M., Fang, M., Aoki, K., Alvarez-Manilla, G., Moremen, K.W., York, W.S., Tiemeyer, M., Pierce, M., Dalton, S., Wells, L.: IDAWG: metabolic incorporation of stable isotope labels for quantitative glycomics of cultured cells. J. Proteome Res. 8, 3816-3823 (2009)

38. Breidenbach, M.A., Gallagher, J.E.G., King, D.S., Smart, B.P., Wu, P., Bertozzi, C.R.: Targeted metabolic labeling of yeast $N$-glycans with unnatural sugars. Proc. Natl. Acad. Sci. U. S. A. 107, 3988-3993 (2010)

39. Zhang, W., Wang, H., Tang, H., Yang, P.: Endoglycosidase-mediated incorporation of ${ }^{18} \mathrm{O}$ into glycans for relative glycan quantitation. Anal. Chem. 83, 4975-4981 (2011)
40. Kuzyk, M.A., Smith, D., Yang, J., Cross, T.J., Jackson, A.M., Hardie, D.B., Anderson, N.L., Borchers, C.H.: Multiple reaction monitoring-based, multiplexed, absolute quantitation of 45 proteins in human plasma. Mol. Cell. Proteomics 8, 1860-1877 (2009)

41. Kim, Y.J., Zaidi-Ainouch, Z., Gallien, S., Domon, B.: Mass spectrometrybased detection and quantification of plasma glycoproteins using selective reaction monitoring. Nat. Protoc. 7, 859-871 (2012)

42. Zhang, H., Wang, Z., Stupak, J., Ghribi, O., Geiger, J.D., Liu, Q.Y., Li, J.: Targeted glycomics by selected reaction monitoring for highly sensitive glycan compositional analysis. Proteomics 12, 2510-2522 (2012)

43. Gil, G.-C., Velander, W.H., Cott, K.E.V.: $N$-glycosylation microheterogeneity and site occupancy of an Asn-X-Cys sequon in plasma-derived and recombinant protein C. Proteomics 9, 2555-2567 (2009)

44. Ahn, Y.H., Lee, J.Y., Lee, J.Y., Kim, Y.-S., Ko, J.H., Yoo, J.S.: Quantitative Analysis of an aberrant glycoform of TIMP1 from colon cancer serum by L-PHA-enrichment and SISCAPA with MRM mass spectrometry. J. Proteome Res. 8, 4216-4224 (2009)

45. Kurogochi, M., Matsushista, T., Amano, M., Furukawa, J.-I., Shinohara, Y., Aoshima, M., Nishimura, S.-I.: Sialic acid-focused quantitative mouse serum glycoproteomics by multiple reaction monitoring assay. Mol. Cell. Proteomics 9, 2354-2368 (2010)

46. Zhao, Y., Wei, J., Wang, J., Ying, W., Zhang, Y., Qian, X.: Fragmentation and site-specific quantification of core fucosylated glycoprotein by multiple reaction monitoring-mass spectrometry. Anal. Chem. 22, 8802-8809 (2011)

47. Song, E., Pyreddy, S., Mechref, Y.: Quantification of glycopeptides by multiple reaction monitoring liquid chromatography/tandem mass spectrometry. Anal. Chem. 26, 1941-1954 (2012)

48. Young, S.P., Stevens, R.D., An, Y., Chen, Y.T., Millington, D.S.: Analysis of a glucose tetrasaccharide elevated in Pompe disease by stable isotope dilution-electrospray ionization tandem mass spectrometry. Anal. Biochem. 316, 175-180 (2003)

49. Hong, Q.T., Ruhaak, L.R., Totten, S.M., Smilowitz, J.T., German, J.B., Lebrilla, C.B.: Label-free absolute quantitation of oligosaccharides using multiple reaction monitoring. Anal. Chem. 86, 2640-2647 (2014)

50. Sowell, J., Wood, T.: Towards a selected reaction monitoring mass spectrometry fingerprint approach for the screening of oligosaccharidoses. Anal. Chim. Acta. 686, 102-106 (2011)

51. Isailovic, D., Kurulugama, R.T., Plasencia, M.D., Stokes, S.T., Kyselova, Z., Goldman, R., Mechref, Y., Novotny, M.V., Clemmer, D.E.: Profiling of human serum glycans associated with liver cancer and cirrhosis by IMSMS. J. Proteome Res. 7, 1109-1117 (2008)

52. Mechref, Y., Kang, P., Novotny, M.V.: Solid-phase permethylation for glycomic analysis. Methods Mol. Biol. 534, 53-64 (2009)

53. Viseux, N., de Hoffmann, E., Domon, B.: Structural analysis of permethylated oligosaccharides by electrospray tandem mass spectrometry. Anal. Chem. 69, 3193-3198 (1997)

54. Mechref, Y., Kang, P., Novotny, M.V.: Differentiating structural isomers of sialylated glycans by matrix-assisted laser desorption/ionization time-offlight/time-of-flight tandem mass spectrometry. Rapid Commun. Mass Spectrom. 20, 1381-1389 (2006)

55. Costello, C.E., Contado-Miller, J.M., Cipollo, J.F.: A glycomics platform for the analysis of permethylated oligosaccharide alditols. J. Am. Chem. Soc. 18, 1799-1812 (2007)

56. Zaia, J.: Mass spectrometry and the emerging field of glycomics. Chem. Biol. 15, 881-892 (2008)

57. Yu, S.-Y., Wu, S.-W., Khoo, K.-H.: Distinctive characteristics of MALDI$\mathrm{Q} / \mathrm{TOF}$ and TOF/TOF tandem mass spectrometry for sequencing of permethylated complex type $N$-glycans. Glycoconj. J. 23, 355-369 (2006)

58. Hu, Y., Mechref, Y.: Comparing MALDI-MS, RP-LC-MALDI-MS, and RP-LC-ESI-MS glycomic profiles of permethylated $\mathrm{N}$-glycans derived from model glycoproteins and human blood serum. Electrophoresis 33, $1768-1777$ (2012)

59. Hu, Y., Zhou, S., Khalil, S.I., Renteria, C.L., Mechref, Y.: Glycomic profiling of tissue sections by LC-MS. Anal. Chem. 85, $4074-4079$ (2013). ASAP

60. Picotti, P., Rinner, O., Stallmach, R., Dautel, F., Farrah, T., Domon, B., Wenschuh, H., Aebersold, R.: High-throughput generation of selected reactionmonitoring assays for proteins and proteomes. Nat. Methods 7, 43-46 (2010)

61. DeSantos-Garcia, J.L., Khalil, S.I., Hussein, A., Mechref, Y.: Enhanced sensitivity of LC-MS analysis of permethylated $N$-glycans through online purification. Electrophoresis 32, 3516-3525 (2011) 